\title{
Cardiovascular Risk Factors and Secondary Events Among Acute and Chronic Stable Myocardial Infarction Patients: Findings from a Managed Care Database
}

Lori D. Bash · Kellee White - Mehul D. Patel · Jinan Liu •

Panagiotis Mavros · Kenneth W. Mahaffey

Received: May 31, 2019 / Published online: August 20, 2019

(C) The Author(s) 2019

\section{ABSTRACT}

Introduction: Long-term risk for recurrent cardiovascular events among myocardial infarction (MI) patients in the acute versus chronic stable phase is not well characterized. This study was conducted to evaluate risk factors associated with all-cause mortality and cardiovascular (CVD) morbidity and to determine the transition period from the acute to chronic stable phase of disease.

Methods: Administrative claims data from a managed care database (2007-2012) were linked

Enhanced Digital Features To view enhanced digital features for this article go to https://doi.org/10.6084/ m9.figshare.9273404.

Electronic supplementary material The online version of this article (https://doi.org/10.1007/s40119019-00147-5) contains supplementary material, which is available to authorized users.

L. D. Bash $(\bowtie)$

Merck \& Co., Inc., Kenilworth, NJ, USA

e-mail: lori_bash@merck.com

K. White

Department of Health Services Administration, University of Maryland College Park School of

Public Health, Maryland, USA

M. D. Patel

Department of Emergency Medicine, School of Medicine, University of North Carolina at Chapel Hill, Chapel Hill, NC, USA to the Social Security Death Index. Kaplan-Meier curves were generated over a 3-year period. The association between risk factors and clinical endpoints was assessed using Cox proportional hazard models. Poisson models estimated the 'transition time' from acute to chronic phase of disease.

Results: On average, recurrent cardiovascular event rates were higher among acute MI patients in comparison to the chronic MI patients during the first 3 months of follow-up. Over the 3-year follow-up period, survival curves became parallel and for some outcomes (i.e., acute myocardial infarction and bleeding events), were not statistically significantly different between the two groups. In both the acute and chronic MI cohorts, diabetes, heart failure, and renal disease were consistently statistically significant and positively associated with greater risk of death and ischemic events. PAD was consistently associated with increased

\section{J. Liu · P. Mavros}

The Janssen Pharmaceutical Companies of Johnson \& Johnson, Titusville, NJ, USA

K. W. Mahaffey

Department of Medicine, Stanford Center for Clinical Research, Stanford University School of Medicine, Stanford, CA, USA 
risk among the chronic cohort and composite endpoints among the acute patients.

Conclusions: Greater understanding of differences in the CVD risk profiles and the transition from acute to chronic stable phase may help identify high-risk patients and inform clinical risk stratification and long-term disease management in MI patients.

Funding: Merck \& Co., Inc., Kenilworth, NJ, USA.

Keywords: Acute coronary syndrome; Mortality; Myocardial infarction; Stable ischemic heart disease

\section{INTRODUCTION}

Patients experiencing myocardial infarctions (MI) are at risk of subsequent cardiovascular events [1-5] and prevention of recurrent events is a major clinical and public health priority. Although their risk is still elevated, the decreasing risk that patients experience over time following an acute event is well known $[6,7]$. However, the timing and implications of the transition between the acute and a chronic stable phase is not well understood.

Atherosclerotic vascular disease can be characterized by two distinct phases: acute and chronic. The acute phase is an early phase of risk after the clinical presentation of acute coronary syndrome (ACS) whereas the chronic phase sets in later and is characterized by the absence or stability of symptoms [8]. Recent treatment guidelines do not define when a patient enters the chronic stable phase. Differentiating between acute and chronic disease conditions may provide insight for the development of therapeutic strategies and enhance decision-making for secondary prevention and long-term disease management among MI patients.

There is evidence to suggest that the pattern of recurrent cardiovascular events of acute patients and those reaching the chronic stable phase differ. For example, Mahaffey et al. found elevated risks of cardiovascular disease mortality, MI, stroke, and bleeding events among acute-phase participants in comparison to chronic-phase participants [8]. The data from this study only included up to 6 months of follow-up for primary endpoints. Risk profiles are not known for longer follow-up periods or outside of a tightly controlled trial setting. Further, studies comparing long-term risk factors and clinical endpoints between acute- and stable-phase MI patients are limited. Thus, additional research is warranted to better understand long-term risks for recurrent and secondary events following acute MI in a realworld setting.

Using administrative claims data from a large, commercially insured population, we characterize and compare associations of cardiovascular risk factors with clinical endpoints (i.e., ischemic events, bleeding events, and allcause mortality) between acute and chronic phase MI patients. Leveraging data from electronic medical records, we also investigated the transition from the acute to chronic phase of the disease process among patients with history of MI using 3 years of follow-up data to complement findings observed in a randomized controlled trial (RCT) setting.

\section{METHODS}

\section{Study Design and Patients}

This retrospective cohort study utilized data from a sample of patients with health care records available from the Optum Clinformatics Data Mart database (OptumInsight, Eden Prairie, MN, USA). This database contains longitudinal enrollment information, inpatient and outpatient services, and records (i.e., medical claims, pharmacy claims, and laboratory data) of over 12 million beneficiaries throughout the US affiliated with a national managedcare network. The commercial health plans are geographically diverse, with the largest proportion of patients found in the South and the Midwest regions. The de-identified Optum database was linked to the Social Security Administration Death Master File to obtain dates of any cause of death for deceased study patients through September 2011. Only allcause death was available. 
Eligible patients $(N=23,352)$ included those having experienced a hospitalization for MI from October 1, 2006 to September 30, 2009, the index period in which a patient experienced an acute MI, or for which there was a documented history of recent MI (Supplementary Figure 1). We created two patient cohorts to mimic the acute and chronic phase following an MI. The acute cohort was defined as patients who had an inpatient medical claim with a primary International Classification of Disease 9th revision (ICD-9) diagnosis of 410.xx occurring within the index period. The index date was set to the date of hospital discharge, and if a patient experienced more than one MI, the index event was defined as the first observed discharge. The chronic MI cohort was selected based on a patient having had a prior hospitalization for acute MI (i.e., inpatient medical claim with 410.xx as the primary diagnosis code) during the index period, followed by a health care encounter (i.e., non-inpatient, any medical claim) at least 2 weeks, but no more than 12 months later (in this way, we created a chronic cohort consistent with the inclusion criteria of the Thrombin Receptor Antagonist in Secondary Prevention of Atherothrombotic Ischemic Events (TRA-2P)-Thrombolysis in Myocardial Infarction (TIMI) 50 trial [6]). The first observed non-MI visit within the index period meeting the requirement for recent history of acute MI was selected, and the index date was set to the date of that visit. Experientially, following an MI event, patients will at first be acute for some period of time, and then hopefully progress to the chronic phase of disease after they stabilize. In that way, every chronic MI patient would have previously been an acute patient for some period. While several chronic patients were at one point "acute patients" (based on these definitions, at the time following their index MI), for these analyses, we forced mutual exclusivity between patient cohorts in order to avoid overlap between acute and chronic MI cohorts. Among the patients meeting the criteria for acute MI $(n=23,352)$, we randomly selected half to ultimately be included in the acute MI cohort $(N=11,676)$, forcing mutually exclusive patient groups (while this isn't necessary for this exercise in comparing different patient experiences during different spectrums of disease, with more than sufficient sample size, it simplifies analyses since no hypotheses are related to actual or relative size of the cohorts, simply disease trends within each. Sensitivity analyses without this exclusion were also done, confirming similar findings). The remaining 50\% were candidates for the chronic MI cohort, along with others meeting chronic inclusion criteria $(N=13,931)$.

Patients were at least 18 years old at the index event and continuously enrolled in the database at least 1 year prior to the index event ("baseline period"). Patients were excluded if they had a medical claim history with diagnosis of stroke, transient ischemic attack (TIA), or bleeding disorders or a pharmacy claim for anticoagulant therapy (Supplementary Figure 2). Patients in both cohorts were followed until September 30, 2011, to ensure a minimum follow-up period of 2 years.

\section{Endpoints}

The following five individual and composite clinical endpoints were assessed as outcomes: (1) all-cause death; (2) all-cause death, acute myocardial infarction (AMI), stroke, or coronary revascularization; (3) all-cause death, AMI, or stroke; (4) AMI; and (5) bleeding events. The clinical endpoints were defined by inpatient primary diagnosis or procedure codes.

\section{Covariates}

Covariates of interest included patient age (defined at the index date), sex (male, female), and health insurance benefit plan (health maintenance organization [HMO], preferred provider organization/exclusive provider organization [PPO/EPO], point-of-service [POS], individual, or other). History of hypertension, hyperlipidemia, diabetes mellitus, MI, coronary artery disease (CAD), angina, heart failure, atrial fibrillation (AF), renal disease, peripheral artery disease, coronary revascularization procedures (e.g., percutaneous coronary intervention [PCI] and coronary artery bypass grafting [CABG], 
and medication use (e.g., antihypertensives, statins, and antiplatelet therapy [APT])) during the baseline period (12-month period prior to index event) were defined using medical and pharmacy claims data.

\section{Statistical Analysis}

Descriptive statistics including frequencies and percentages for categorical variables and mean and standard deviations for continuous variables were presented by phase of disease. Follow-up time was calculated from baseline to the earliest date in which a patient experienced an outcome. Follow-up times were censored at the end of enrollment or end of study period. For endpoints not including death, follow-up time was censored at date of death. To compare longterm risks between acute and chronic cohorts, the unadjusted incidence rate and 95\% confidence intervals (CIs) of each outcome were estimated with Poisson regression models. Cumulative incidences ("risk" $=$ number of events divided by number of patients) were also calculated. Kaplan-Meier methods were used to construct unadjusted survival curves for each of the outcomes over 3 years and log-rank tests were performed to evaluate differences between cohorts for each of the events. A minimally adjusted (age and gender) incidence rate was plotted over time in a fifth-order polynomial Poisson model to visualize the 'transition time' from acute to chronic phase of disease.

Separate Cox proportional hazards regression models estimated patient characteristics associated with risk of the aforementioned five outcomes. We adjusted for age, sex, benefit plan type, comorbidities, and prior APT use. These covariates were selected a priori based on the literature and were retained in the model regardless of statistical significance. A $p$ value of $\leq 0.05$ was considered to be statistically significant. Analyses were performed using SAS 9.4 (Cary, NC, USA).

We conducted sensitivity analyses to examine whether results changed when the chronic MI cohort definition in the primary analysis was varied for greater specificity. We compared alternative definitions for chronic MI patients by narrowing the time window required ( 2 weeks-12 months) between the AMI and outpatient visit using the following windows: 1-12 months; 2-12 months; 3-12 months; and 6-12 months. Patient characteristics, outcome rates, and survival curves were examined for each of these definitions and compared with the time frame in the primary analysis.

\section{Compliance with Ethics Guidelines}

This study does not use data from animals, human specimens, or human participants and did not contain any identifiable protected health information. This de-identified secondary observational analysis was deemed exempt by the Institutional Review Board. Informed consent was obtained from all individual participants included in the study.

\section{RESULTS}

The final study sample yielded a total of 14,817 participants (acute MI: $N=6645$; and chronic MI: $N=8172$ ). Baseline patient demographic and clinical characteristics are presented in Table 1. Overall, the two cohorts exhibited a similar distribution in age, sex, and health benefit plan type. Fewer patients in the acute MI cohort reported a history of hypertension, hyperlipidemia, heart failure, angina, CAD, revascularization procedures, and medication use.

Table 2 shows incidence rates by acute and chronic MI cohort status for all-cause death, composite ischemic events, and bleeding events. Incidence rates across all endpoints were higher in the acute MI cohort. The unadjusted 3-year Kaplan-Meier curves are shown for acute and chronic MI patient cohorts by the five outcomes (Fig. 1). For each outcome, similar curves were observed for the acute and chronic MI cohorts, with acute MI patients, demonstrating overall higher event rates. A divergence in the acute versus chronic MI curves is apparent in the first month of follow-up for all of the outcomes (all log-rank $p<0.0001$, except for AMI with $p=0.0005)$, with the exception of bleeding events $(p=0.2350)$. 
Table 1 Patient demographic and medical history by MI cohort among Optum database participants, 2006-2009

\begin{tabular}{|c|c|c|}
\hline & $\begin{array}{l}\text { Acute } \\
\text { MI cohort } \\
(N=6645) \\
\text { Number }(\%)\end{array}$ & $\begin{array}{l}\text { Chronic } \\
\text { MI cohort } \\
(N=8172) \\
\text { Number }(\%)\end{array}$ \\
\hline Age, mean (SD) & $57.57(11.0)$ & $56.91(10.7)$ \\
\hline
\end{tabular}

Age group

$\begin{array}{lcc}<45 & 672(10.1) & 864(10.6 \%) \\ 45-54 & 1995(30.0) & 2580(31.6) \\ 55-64 & 2571(38.7) & 3172(38.8) \\ 65-74 & 811(12.2) & 921(11.3) \\ \geq 75 & 596(9.0) & 635(7.8) \\ \text { Sex } & & \\ \text { Female } & 1774(26.7) & 2083(25.5)\end{array}$

Benefit plan type

$\begin{array}{lcc}\text { HMO } & 649(9.8) & 912(11.2) \\ \text { PPO/EPO } & 1389(20.9) & 1631(20.0) \\ \text { POS } & 4106(61.8) & 5095(62.4) \\ \text { Individual } & 470(7.1) & 499(6.1) \\ \text { Other } & 31(0.47) & 35(0.43) \\ \text { Medical history } & & \\ \text { Hypertension }{ }^{a} & 4649(70.0) & 7665(93.8) \\ \text { Hyperlipidemia } & 4426(66.6) & 7660(93.7) \\ \text { Diabetes } & 1862(28.0) & 2449(30.0) \\ \text { MI } & 226(3.4) & 8172(100.0) \\ \text { Coronary } & 205(3.1) & 7652(93.6) \\ \quad \text { revascularization } \\ \quad \text { prior to index date) }\end{array}$

Table 1 continued

\begin{tabular}{lcl}
\hline & $\begin{array}{l}\text { Acute } \\
\text { MI cohort } \\
(\boldsymbol{N}=\mathbf{6 6 4 5}) \\
\text { Number }(\%)\end{array}$ & $\begin{array}{l}\text { Chronic } \\
\text { MI cohort } \\
(\boldsymbol{N}=\mathbf{8 1 7 2}) \\
\text { Number }(\%)\end{array}$ \\
\hline CAD & $5750(86.5)$ & $7934(97.1)$ \\
Angina & $812(12.2)$ & $1407(17.2)$ \\
Heart failure & $1218(18.3)$ & $1752(21.4)$ \\
AF & $461(6.9)$ & $625(7.6)$ \\
Renal disease & $634(9.5)$ & $743(9.2)$ \\
PAD & $447(6.7)$ & $639(7.8)$ \\
Medication history & & \\
Antihypertensives & $2885(43.4)$ & $7361(90.2)$ \\
Statins & $1911(28.8)$ & $6480(79.3)$ \\
Antiplatelets & $617(9.3)$ & $6421(78.6)$ \\
\hline
\end{tabular}

$H M O$ health maintenance organization, $P P O$ preferred provider organization, EPO exclusive provider organization, $P O S$ point-of-service, $M I$ myocardial infarction, $P C I$ percutaneous coronary intervention, $C A B G$ coronary artery bypass grafting, $C A D$ coronary artery disease, $A F$ atrial fibrillation, $P A D$ peripheral artery disease

${ }^{a}$ Positive for hypertension if evidence of diagnosis or antihypertensive medication use; not counted if heart failure patient on antihypertensive but no hypertension diagnosis

b Positive for hyperlipidemia if evidence of diagnosis or statin medication use

Kaplan-Meier failure probability estimates based on the failure curves indicated that acute MI patients have higher risk than chronic MI patients for the all-cause death, AMI, stroke, coronary revascularization composite outcome (Fig. 1c). Although there is an early increased risk observed for the acute MI cohort in comparison to the chronic cohort for death (Fig. 1a), AMI (Fig. 1b), and death, AMI, stroke (Fig. 1d), there were no statistically significant differences in the curves through year three. Varying the window for chronic MI (e.g., ranging from 1-12 months to 6-12 months) resulted in lower failure rates for death and the composite ischemic outcomes as the definition increased in specificity (Fig. 1a, c, d). The opposite was 
Table 2 Outcome incidence rates in acute and chronic MI cohorts

\begin{tabular}{|c|c|c|c|c|c|}
\hline & \multirow[t]{2}{*}{ Death } & \multicolumn{3}{|c|}{ Composite ischemic events } & \multirow{2}{*}{$\begin{array}{l}\text { Bleeding } \\
\text { events }\end{array}$} \\
\hline & & $\begin{array}{l}\text { Death/AMI/stroke/ } \\
\text { revascularization }\end{array}$ & $\begin{array}{l}\text { Death/ } \\
\text { AMI/stroke }\end{array}$ & AMI & \\
\hline \multicolumn{6}{|l|}{ Acute MI cohort $(N=6645)$} \\
\hline Number of events & 403 & 1576 & 834 & 399 & 75 \\
\hline Total person-time (in years) & $11,317.68$ & 9534.7 & $10,712.3$ & $10,802.17$ & 11,232 \\
\hline $\begin{array}{l}\text { Incidence rate per } 1000 \text { person- } \\
\text { years }(95 \% \mathrm{CI})\end{array}$ & $\begin{array}{l}35.6(32.29 \\
39.26)\end{array}$ & $165.3(157.33,173.66)$ & $\begin{array}{l}77.9(72.75 \\
83.32)\end{array}$ & $\begin{array}{l}36.9(33.49 \\
40.75)\end{array}$ & $\begin{array}{c}6.7(5.33, \\
8.37)\end{array}$ \\
\hline Cumulative incidence & $6.1 \%$ & $23.7 \%$ & $12.6 \%$ & $6.0 \%$ & $1.1 \%$ \\
\hline \multicolumn{6}{|l|}{ Time to event (days), $n$} \\
\hline $1-7$ & 29 & 138 & 86 & 50 & 2 \\
\hline $8-30$ & 109 & 330 & 145 & 30 & 8 \\
\hline $31-180$ & 105 & 477 & 199 & 84 & 16 \\
\hline $181-365$ & 52 & 239 & 126 & 72 & 17 \\
\hline$\geq 365$ & 108 & 392 & 278 & 163 & 32 \\
\hline \multicolumn{6}{|l|}{ Chronic MI cohort $(N=8172)$} \\
\hline Number of events & 324 & 1462 & 762 & 388 & 74 \\
\hline Total person-time (in years) & $13,533.48$ & $11,818.5$ & $12,907.9$ & $13,023.6$ & $13,448.9$ \\
\hline $\begin{array}{l}\text { Incidence rate per } 1000 \text { person- } \\
\text { years }(95 \% \mathrm{CI})\end{array}$ & $\begin{array}{l}23.9(21.47 \\
26.69)\end{array}$ & $123.7(117.52,130.21)$ & $\begin{array}{l}59.0(54.99 \\
63.38)\end{array}$ & $\begin{array}{l}29.8(26.97 \\
32.91)\end{array}$ & $\begin{array}{c}5.5(4.38 \\
6.91)\end{array}$ \\
\hline Cumulative incidence & $4.0 \%$ & $17.9 \%$ & $9.3 \%$ & $4.6 \%$ & $0.91 \%$ \\
\hline \multicolumn{6}{|l|}{ Time to event (days), $n$} \\
\hline $1-7$ & 3 & 73 & 15 & 11 & 4 \\
\hline $8-30$ & 16 & 155 & 35 & 20 & 6 \\
\hline $31-180$ & 96 & 451 & 188 & 73 & 19 \\
\hline $181-365$ & 58 & 285 & 157 & 77 & 15 \\
\hline$\geq 365$ & 151 & 498 & 367 & 207 & 30 \\
\hline
\end{tabular}

true for AMI alone (Fig. 1b, the narrowest definition of 'chronic' showed higher failure rates at 3 years).

In age- and gender-adjusted polynomial Poisson models, all-cause death rates plateau between 3 and 4 months for acute and chronic MI cohort patients, when the risk of events of the cohorts starts to become similar (plotted in
Fig. 2, see Supplemental Table S1 for specific estimates). Similar patterns were observed for men and women and for the death, AMI, and stroke composite outcome.

The direction and magnitude of association between select risk factors were similar across outcomes for acute and chronic MI patients (Table 3). In both groups, older age ( $\geq 65$ ), heart 
failure, and renal disease were more strongly associated with risk of death compared to the ischemic endpoints. Known risk factors including history of diabetes, heart failure, renal disease, and PAD were statistically significantly associated with increased risk for all endpoints (all-cause death, MI, and composites) among the chronic cohort. In contrast, while the same trend was true for diabetes, heart failure, and renal disease among the acute cohort, observations were not as consistent with PAD, which wasn't associated with death or AMI as individual endpoints, but only statistically significantly associated with increased risk of composite endpoints among the acute patients. Hyperlipidemia was negatively associated with death and composite ischemic endpoints in both cohorts. In the acute MI cohort, prior APT use was associated with increased risk of three composite ischemic endpoints but among chronic MI patients, it was associated with decreased risk of death and the composite endpoint of death, AMI, and stroke. However, prior APT use was also associated with a marginally significant increased risk of the composite ischemic endpoint for death, AMI, stroke, coronary revascularization.

\section{DISCUSSION}

Using a large administrative claims database, we analyzed data from MI patients during the acute and chronic phase of their disease with up to 3 years of follow-up. Our findings suggest some similarities between those patients in the acute and the chronic phase, but also some important differences in cardiovascular risk profiles and patterns of clinical outcomes. Further, our analyses suggest that acute patients may transition to the chronic stable phase around 3-4 months after which the pattern of outcomes is similar, though risk differences remain. When acute and chronic cohorts are followed for up to 3 years, there is an observable difference between their survival curves, which is suggestive of convergence over time.

The short-term prognosis following MI among acute patients is worse than those who reach the chronic stable phase of atherosclerotic disease. This finding is consistent with several recent studies demonstrating higher acute risk versus lower long-term risk [8, 19-22]. However, few studies have reported the risk factors associated with relatively long-term clinical endpoints between acute and chronic stable MI patients $[9,10]$. Our results are consistent with Jernberg et al., which showed similarities in overall risk after 3 years of follow-up between the acute and stable MI patients [10].

These overall patterns for clinical endpoints are consistent with the evidence from RCT populations at 6 months of follow-up. In Mahaffey et al., the composite endpoint of vascular death was combined with $\mathrm{MI}$ and stroke, and an increased risk of death was observed between the patients in the acute and the chronic phase [8]. Our data show a similar pattern for outcomes that include death and ischemic endpoints. The magnitude of risk varies for each outcome, where the increased risk among the acute group is most pronounced for death. When comparing composite endpoints comprising death and different combinations of ischemic outcomes (e.g., death, AMI, and stoke), our findings suggest that acute patients transition to the chronic phase around 3-4 months after the index MI.

At baseline, patients in the acute phase had less severe risk factor profiles as indicated by lower prevalence of hypertension, hyperlipidemia, and angina, than those in the chronic stable phase. This is likely a result of increased diagnoses of these comorbidities once patients have additional contact with healthcare providers, following an acute event, and increased documentation of these diagnoses. In general, clinical factors associated with cohort-specific outcomes and clinical endpoints, not unexpectedly, overlapped. In both cohorts, several traditional clinical risk factors were associated with greater risk of death and ischemic events. This finding is consistent with a recent study showing that conventional cardiovascular risk factors were not markedly different between stable and acute MI populations [10]. These similarities may underscore the complex interactions of underlying pathophysiologic processes and may point to biomarkers that may be more important for distinguishing the phases of 
(A) All-cause death, logrank p.00.0001
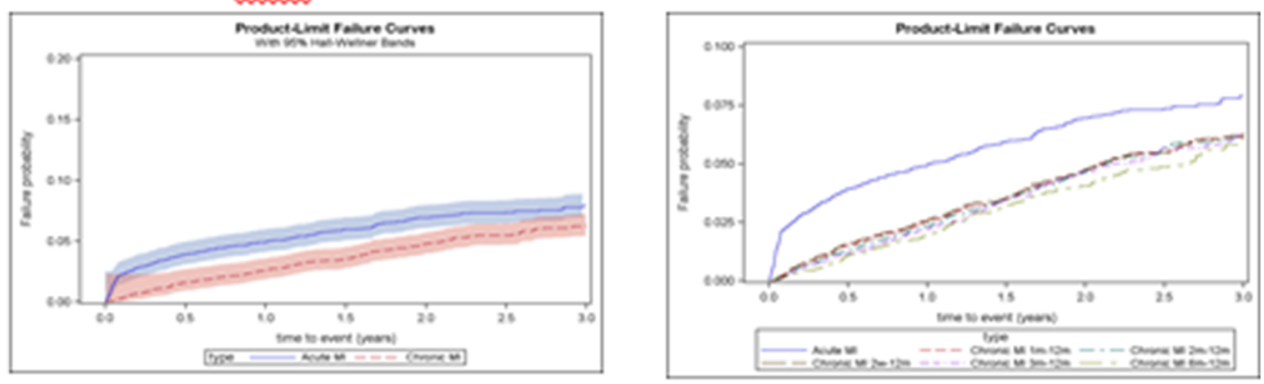

(B) AMl, logrank, $p=0.0005$
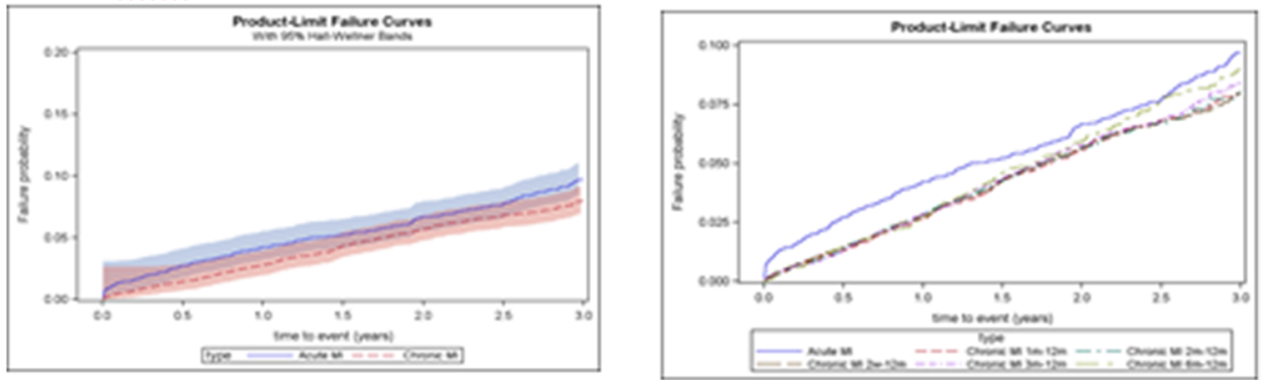

(C) Death/AMI/Stroke/Coronary Revascularization, losrank p $\$ 0.0001$
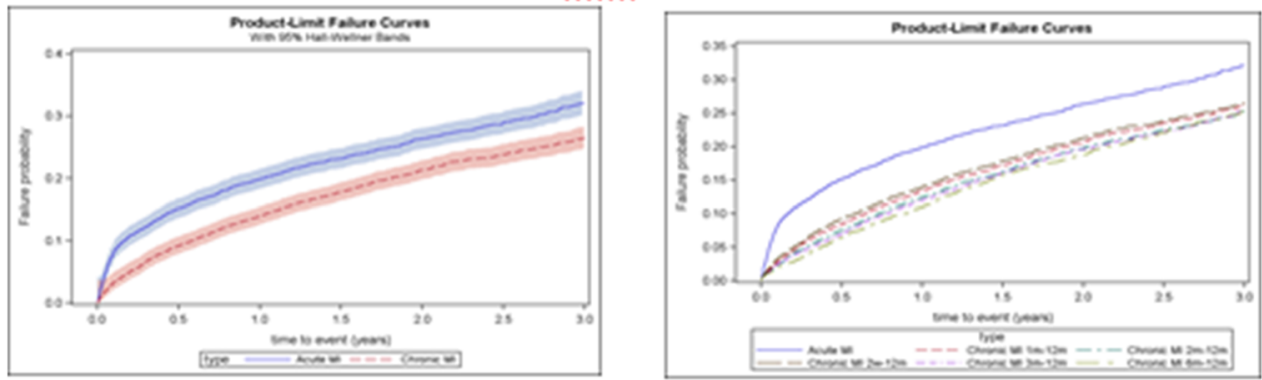

(D) Death/AMUStroke, logrank p\$0.0001
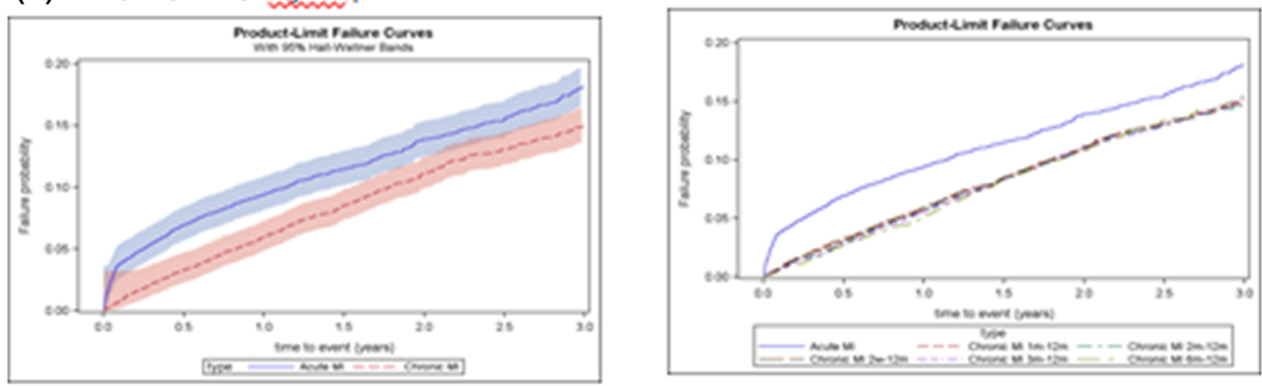

(E) Bleeding Events, logrank $p=0.2350$
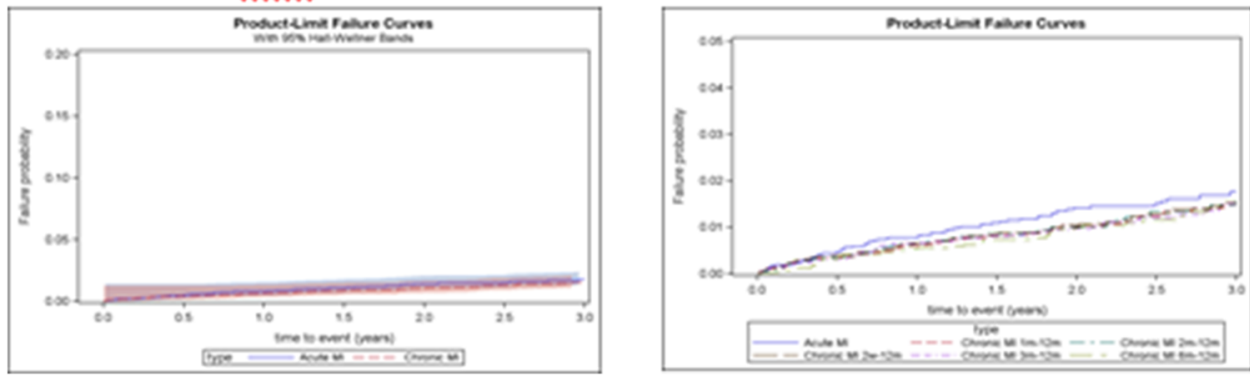
4Fig. 1 Kaplan-Meier curves over 3 years in acute and chronic MI cohorts for: a death, b AMI, c death, AMI, stroke, and coronary revascularization, $\mathbf{d}$ death, AMI, and stroke, and e bleeding events. For each outcome, Kaplan-Meier curves for the chronic MI with varying windows (2 weeks-12 months; 1 month-12 months; 2 months-12 months; 3 months-12 months; 6 months12 months). AMI: acute myocardial infarction

disease. Recent studies have identified inflammatory markers, fibrinolytic and hemostatic factors as potential biomarkers [11]. Future research is needed to determine if these biomarkers have discriminatory capacity between groups.
However, there were some pronounced differences in the association of key risk factors by MI cohort status. Angina was only associated with greater risk of ischemic endpoints and bleeding events in the chronic MI patients. Prior APT use, while associated with a reduced risk of death and the combined ischemic endpoint among the chronic MI patients, was also associated with an increased risk of ischemic events in the acute MI cohort. These results are generally inconsistent with prior research demonstrating the effectiveness of APT therapy in reducing the risk of major adverse cardiovascular events in patients with either acute or stable disease [6, 12-14]. Some studies have found an increased risk of bleeding and

(A) Death

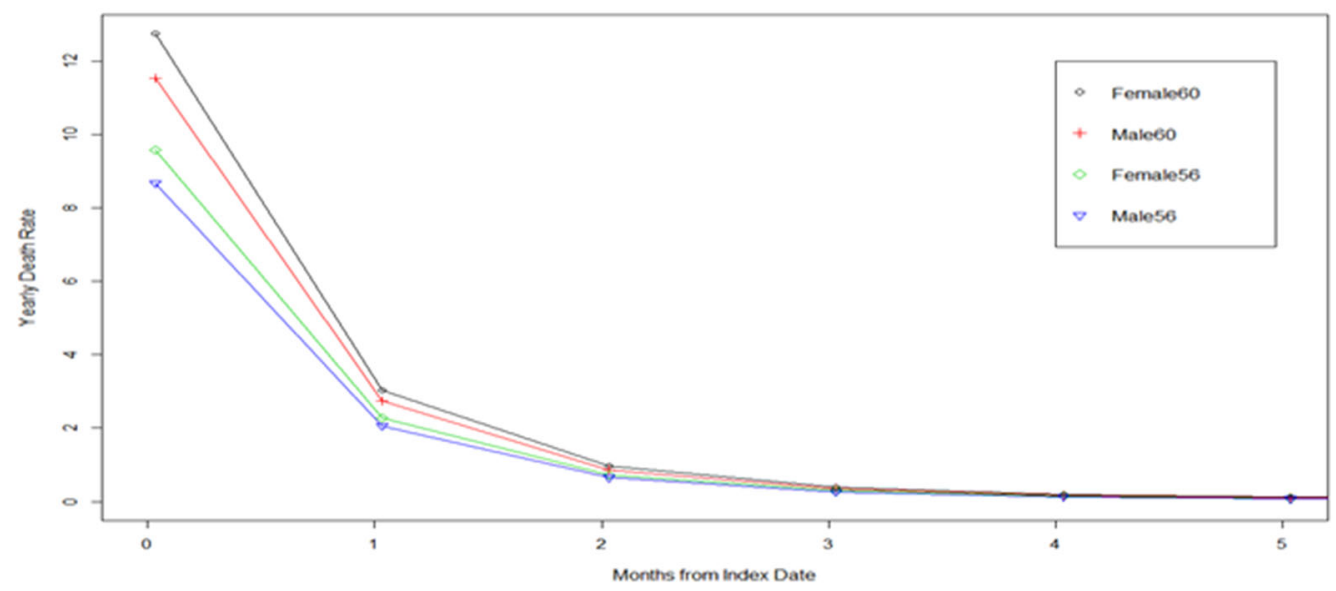

(B) Death, AMI, Stroke

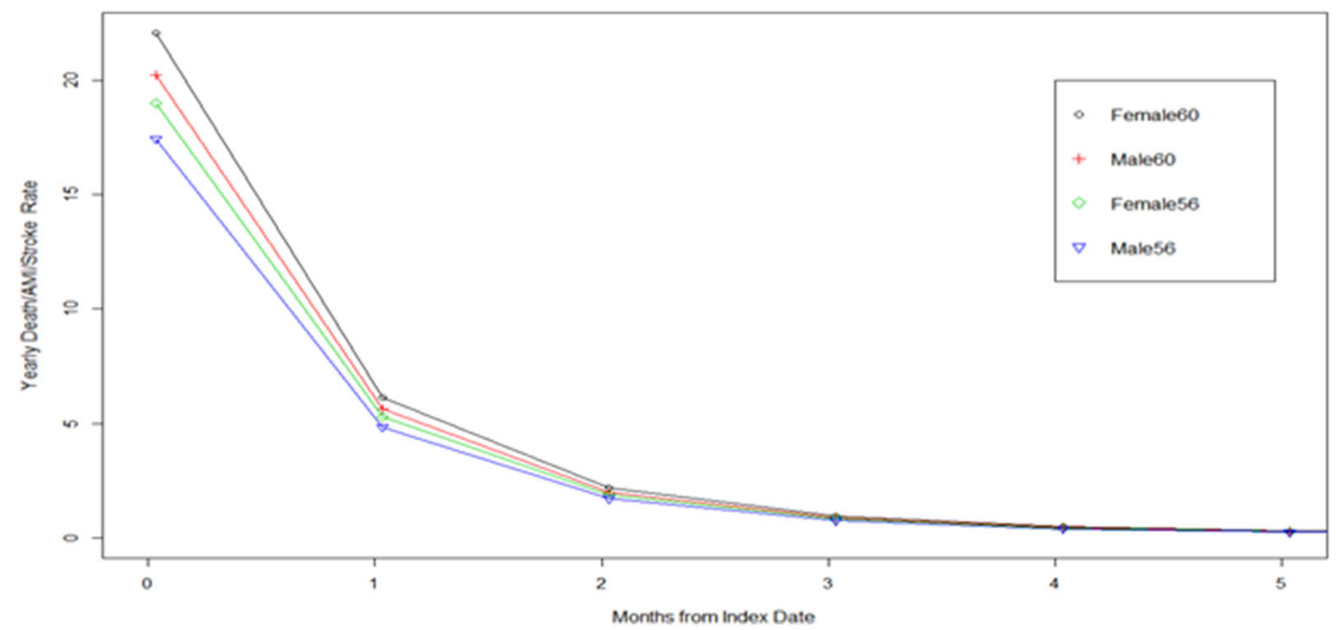

Fig. 2 Adjusted yearly incidence rate of $\mathbf{a}$ death and $\mathbf{b}$ death, AMI, and stroke, over time 


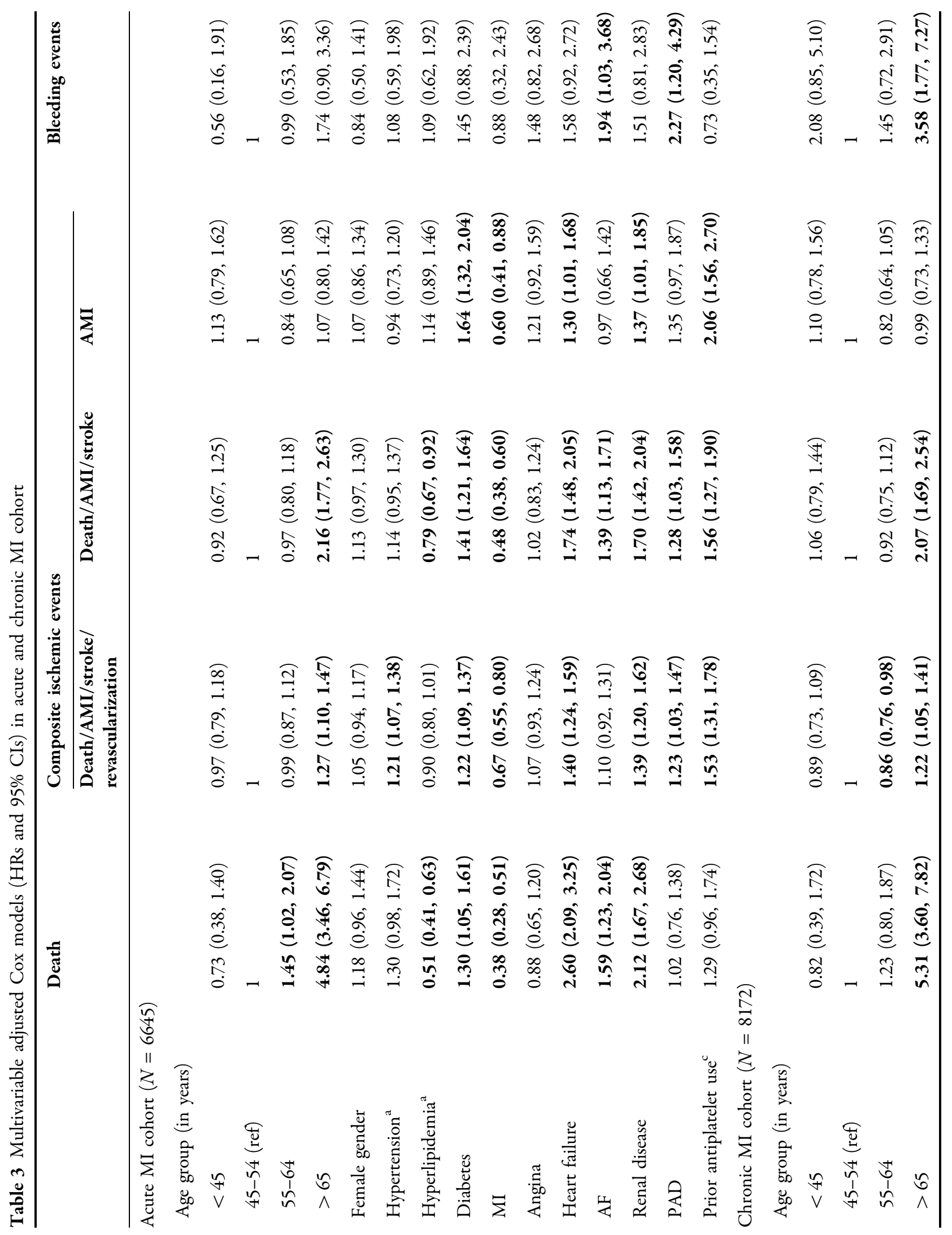




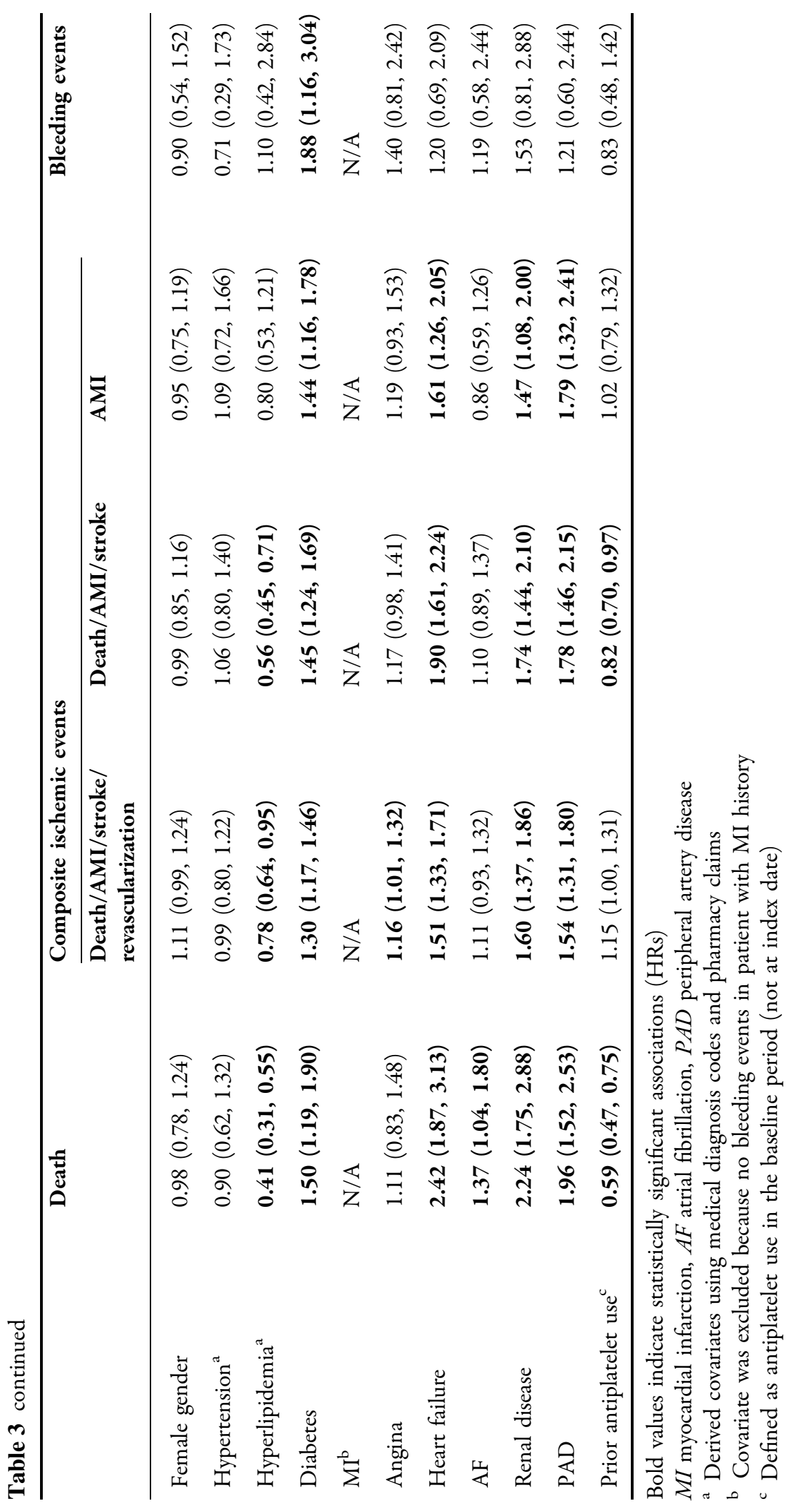


ischemic events in specific patient sub-groups such as those with stroke [6, 15-17]. In our sample, individuals who had a medical claims history of a stroke or TIA diagnosis were excluded from the analytic sample and so we do not think that stroke prevalence may have accounted for the different association in APT use between the two cohorts. Moreover, acute patients are at a higher risk of ischemic events [18] and are more likely to use APTs. The protective association observed among chronic MI patients may in part be attributable to tolerance to continued APT use over time. It is also possible that confounding by indication may account for these observations since APT use was measured at baseline and not at follow-up. Limitations in the detail available on both APT and cause of death cannot be dismissed.

We compared four alternative time frames for defining the chronic phase and our data suggest that this can impact the pattern and shape of survival curves. The all-cause death and composite event rates decreased as the window defining the chronic phase was more restrictive (and the definition of "chronic" more specific and distinct from acute). Using a lower bound of 2 weeks may still include (at least some) acute MI patients, whereas using 6 months may lead to the selection of healthier survivors. Interestingly, the opposite was true in looking at AMI event rates; for this outcome, all chronic cohorts had similar failure rates for the first 1.5-2 years before beginning to diverge from one another. Over the 3-year follow-up period, the most restrictive definition of chronic patients had the highest failure rate (highest probability of experiencing another MI event), approaching the rate of the acute MI cohort. This observation, that those who were more likely to survive were more likely to experience MI, may implicate other competing risks of death, and the limitations in our data in not having cause specific death data available. In sensitivity analyses, the multivariable analyses were robust to the different definitions of chronic MI (data not shown). While it remains unclear how to define an MI patient as chronic stable relying only on administrative claims data, the window for "recent" acute MI used may influence findings. We observed a relatively finite window of time $(\sim 3-4$ months after the index MI), in which the change in risk over time occurs and a patient may progress between phases in the disease process.

To our knowledge, this is one of the first studies to characterize and compare outcome patterns with 3 years of follow-up, between acute and chronic MI patients using a longitudinal administrative claims database with linked inpatient, outpatient, and pharmacy data. However, the results of the study need to be interpreted in light of several limitations. The claims database relies on coding to define patients and clinical events. Clinical comorbidities and medical history not captured or inaccurately recorded in claims data could have impacted baseline clinical differences observed between the acute or chronic MI cohort. In addition, non-prescription medications may not be fully captured and use of prescription medications is based upon outpatient pharmacy prescription fills. There is a lack of cause-specific mortality data, leaving competing risks of death unrelated to acute/chronic status unaccounted. Similar to prior clinical trial protocol [6], patients were characterized in the chronic patient cohort if an event occurred within the previous 2 weeks- 12 months. A definitive recommendation or consensus regarding the point at which the patient will transition into the chronic phase has not been established and remains largely a subjective measure which might not be truly ascertainable through claims data. Further, we did not have information on confounding variables such as smoking and socioeconomic status, which may lead to residual confounding. While we caution generalizing results to other populations not represented by this managed care database that may vary in patient characteristics and treatment patterns, our findings are still relevant since the limitations were inherent to both acute and stable MI patient groups.

\section{CONCLUSIONS}

In this study, we sought to characterize longterm risk profiles of acute and chronic stable MI patients and compare several definitions of 
chronic MI to improve management of these conditions. This study extends information on the long-term survival and cardiovascular risk profile of acute and chronic MI patients using data from a large administrative claims database. The short-term survival and cardiovascular risks in acute and chronic MI patients varied. The acute MI cohort remained at higher risk for death and the composite death/AMI/stroke endpoint in comparison to the chronic MI cohort, although the risks become similar starting around 3-4 months. While prior attempts have been made to infer acute and chronic phase transition, it remains unclear how to define the transition between acute and chronic phases. Our findings provide meaningful insight into the transition from acute to chronic phase, which has implications for postMI prognosis, disease management, and secondary prevention over the long term.

\section{ACKNOWLEDGEMENTS}

We thank the participants of the study.

Funding. Analyses were funded by Merck \& Co., Inc., Kenilworth, NJ, USA. All authors had full access to all of the data in this study and take complete responsibility for the integrity of the data and accuracy of the data analysis. No funding was received for the Rapid Service Fee.

Authorship. All named authors meet the International Committee of Medical Journal Editors (ICMJE) criteria for authorship for this article, take responsibility for the integrity of the work as a whole, and have given their approval for this version to be published.

Author Contributions. Lori D. Bash, Panagiotis Mavros conceived the study and participated in its design, analyses, coordination, and access to data. KW drafted the manuscript and interpretation of results. Mehul D. Patel, Jinan Liu, and Lori D. Bash participated in the statistical analysis, interpretation of results, and manuscript revisions. Kenneth W. Mahaffey and Panagiotis Mavros were involved in the design and interpretation of results. All authors read and approved the final manuscript.

Disclosures. Lori D. Bash was a full time employee of Merck \& Co., Inc., Kenilworth, NJ, USA at the time this work was conducted. Panagiotis Mavros was a full-time employee of Merck \& Co., Inc., Kenilworth, NJ, USA at the time this work was conducted. Mehul D. Patel was a full-time employee of Merck \& Co., Inc., Kenilworth, NJ, USA at the time this work was conducted. Jinan Liu was a full-time employee of Merck \& Co., Inc., Kenilworth, NJ, USA at the time this work was conducted. Kenneth W. Mahaffey received consultation fees from Merck \& Co., Inc., Kenilworth, NJ, USA. Kellee White received consultation fees from Merck \& Co., Inc., Kenilworth, NJ, USA. Kenneth W. Mahaffey is a member of the journal's Editorial Board.

Compliance with Ethics Guidelines. This study does not use data from animals, human specimens, or human participants and did not contain any identifiable protected health information. This de-identified secondary observational analysis was deemed exempt by the Institutional Review Board. Informed consent was obtained from all individual participants included in the study.

Data Availability. The datasets generated and/or analyzed for this study are not publicly available since they include electronic medical records of patients from a secondary source.

Open Access. This article is distributed under the terms of the Creative Commons Attribution-NonCommercial 4.0 International License (http://creativecommons.org/licenses/ by-nc/4.0/), which permits any noncommercial use, distribution, and reproduction in any medium, provided you give appropriate credit to the original author(s) and the source, provide a link to the Creative Commons license, and indicate if changes were made. 


\section{REFERENCES}

1. Bhatt DL, Eagle KA, Ohman EM, Hirsch AT, Goto S, Mahoney EM, et al. Comparative determinants of 4-year cardiovascular event rates in stable outpatients at risk of or with atherothrombosis. JAMA-J Am Med Assoc. 2010;304(12):1350-7.

2. Aliprandi-Costa B, Ranasinghe I, Chow V, Kapila S, Juergens C, Devlin G, et al. Management and outcomes of patients with acute coronary syndromes in Australia and New Zealand, 2000-2007. Med J Aust. 2011;195(3):116-21.

3. Park HW, Yoon $\mathrm{CH}$, Kang $\mathrm{SH}$, Choi DJ, Kim HS, Cho MC, et al. Early- and late-term clinical outcome and their predictors in patients with ST-segment elevation myocardial infarction and non-STsegment elevation myocardial infarction. Int $\mathrm{J}$ Cardiol. 2013;169(4):254-61.

4. Eagle KA, Ginsburg GS, Musunuru K, Aird WC, Balaban RS, Bennett SK, et al. Identifying patients at high risk of a cardiovascular event in the near future current status and future directions: report of a National Heart, Lung, and Blood Institute Working Group. Circulation. 2010;121(12):1447-54.

5. Antman EM, Cohen M, Bernink P, McCabe $\mathrm{CH}$, Horacek T, Papuchis G, et al. The TIMI risk score for unstable angina/non-ST elevation MI-A method for prognostication and therapeutic decision making. JAMA-J Am Med Assoc. 2000;284(7):835-42.

6. Morrow DA, Braunwald E, Bonaca MP, Ameriso SF, Dalby AJ, Fish MP, et al. Vorapaxar in the secondary prevention of atherothrombotic events. N Engl J Med. 2012;366(15):1404-13.

7. Bhatt DL, Fox KAA, Hacke W, Berger PB, Black HR, Boden WE, et al. Clopidogrel and aspirin versus aspirin alone for the prevention of atherothrombotic events. N Engl J Med. 2006;354(16):1706-17.

8. Mahaffey KW, Wojdyla DM, Pieper KS, Tricoci P, Alexander JH, Lincoff AM, et al. Comparison of clinical trial outcome patterns in patients following acute coronary syndromes and in patients with chronic stable atherosclerosis. Clin Cardiol. 2014;37(6):337-42.

9. Alcock RF, Yong ASC, Ng ACC, Chow V, Cheruvu C, Aliprandi-Costa B, et al. Acute coronary syndrome and stable coronary artery disease: are they so different? Long-term outcomes in a contemporary PCI cohort. Int J Cardiol. 2013;167(4):1343-6.

10. Jernberg T, Hasvold $\mathrm{P}$, Henriksson $\mathrm{M}$, Hjelm $\mathrm{H}$, Thuresson M, Janzon M. Cardiovascular risk in post-myocardial infarction patients: nationwide real world data demonstrate the importance of a long-term perspective. Eur Heart J. 2015;36(19):1163-70.

11. Beatty $\mathrm{AL}, \mathrm{Ku} \mathrm{IA}$, Bibbins-Domingo $\mathrm{K}$, Christenson RH, DeFilippi CR, Ganz P, et al. Traditional risk factors versus biomarkers for prediction of secondary events in patients with stable coronary heart disease: from the Heart and Soul Study. J Am Heart Assoc. 2015;4(7):9.

12. Magnani G, Bonaca MP, Braunwald E, Dalby AJ, Fox KA, Murphy SA, et al. Efficacy and safety of vorapaxar as approved for clinical use in the United States. J Am Heart Assoc. 2015;4(3):e001505.

13. Bohula EA, Bonaca MP, Braunwald E, Aylward PE, Corbalan R, De Ferrari GM, et al. Atherothrombotic risk stratification and the efficacy and safety of vorapaxar in patients with stable ischemic heart disease and previous myocardial infarction. Circulation. 2016;134(4):304-13.

14. Baigent C, Sudlow C, Collins R, Peto R, Antithromboci Trialists C. Collaborative metaanalysis of randomised trials of antiplatelet therapy for prevention of death, myocardial infarction, and stroke in high risk patients. $\mathrm{Br}$ Med J. 2002;324(7329):71-86.

15. Ungar L, Clare RM, Rodriguez F, Kolls BJ, Armstrong PW, Aylward P, et al. Stroke outcomes with vorapaxar versus placebo in patients with acute coronary syndromes: insights from the TRACER trial. J Am Heart Assoc. 2018;7(24):e009609.

16. Tricoci P, Huang Z, Held C, Moliterno DJ, Armstrong PW, Van de Werf F, et al. Thrombin-receptor antagonist vorapaxar in acute coronary syndromes. N Engl J Med. 2012;366(1):20-33.

17. Vyas A, Bash LD, Patel MD, Simpson RJ Jr. Changes in treatment patterns and incremental health care utilization due to P2Y12-associated complications in patients with acute coronary syndrome. J Manag Care Spec Pharm. 2017;23(9):947-56.

18. Ibanez B, James S, Agewall S, Antunes MJ, Bucciarelli-Ducci C, Bueno H, et al. 2017 ESC Guidelines for the management of acute myocardial infarction in patients presenting with ST-segment elevation: the Task Force for the management of acute myocardial infarction in patients presenting with ST-segment elevation of the European Society of Cardiology (ESC). Eur Heart J. 2018;39(2):119-77.

19. Choi SY, Kim MH, Serebruany V. Comparison of ACUITY, CRUSADE, and GRACE risk scales for predicting clinical outcomes in patients treated with dual-antiplatelet therapy. TH Open. 2018;2(4):e399-406. 
20. Zafrir B, Adawi S, Khalaily M, Jaffe R, Eitan A, Barnett-Griness O, Saliba W. Long-term risk stratification of patients undergoing coronary angiography according to the thrombolysis in myocardial infarction risk score for secondary prevention. J Am Heart Assoc. 2019;8(14):e012433.

21. Puymirat E, Bonaca M, Fumery M, Tea V, Aissaoui N, Lemesles G, Bonello L, Ducrocq G, Cayla G, Ferrières J, Schiele F, Simon T, Danchin N, FAST-MI investigators. Atherothrombotic risk stratification after acute myocardial infarction: the thrombolysis in myocardial infarction risk score for Secondary Prevention in the light of the French Registry of
Acute ST Elevation or non-ST Elevation Myocardial Infarction registries. Clin Cardiol. 2019;42(2):227-34.

22. Huang D, Cheng YY, Wong YA, Yung SA, Tam CF, Chan KK, Lam CS, Yiu KH, Hai JJ, Lau CP, Chan EW, Chiang CE, Wong KL, Cheung T, Cheung BM, Feng YQ, Tan N, Chen JY, Yue WS, Hu HX, Chen L, Tse HF, Chan PH, Siu CW. Thrombolysis in myocardial infarction risk score for secondary prevention of recurrent cardiovascular events in a realworld cohort of post-acute myocardial infarction patients. Circ J. 2019;83(4):809-17. 